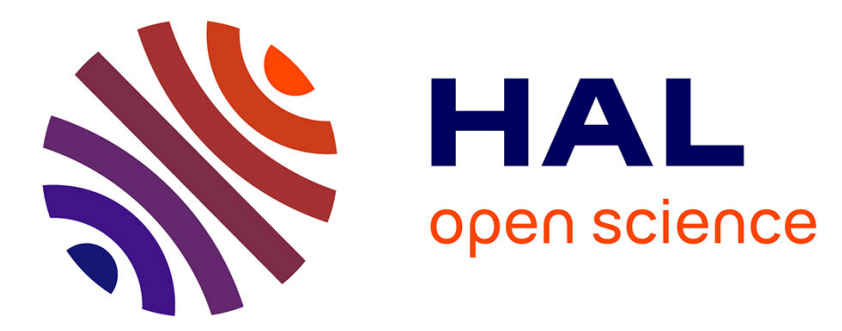

\title{
Novel Perfluorinated Triblock Amphiphilic Copolymers for Lipid-Shelled Microbubble Stabilization
}

Yohann Corvis, Simona Manta, Caroline Thébault, Olivier Couture, Hélène

Dhotel, Jean-Philippe Michel, Johanne Seguin, Michel Michel, Philippe

Espeau, Chantal Pichon, et al.

\section{To cite this version:}

Yohann Corvis, Simona Manta, Caroline Thébault, Olivier Couture, Hélène Dhotel, et al.. Novel Perfluorinated Triblock Amphiphilic Copolymers for Lipid-Shelled Microbubble Stabilization. Langmuir, 2018, 34 (33), pp.9744-9753. 10.1021/acs.langmuir.8b01668 . hal-02123140

\section{HAL Id: hal-02123140 \\ https://hal.science/hal-02123140}

Submitted on 3 Jun 2021

HAL is a multi-disciplinary open access archive for the deposit and dissemination of scientific research documents, whether they are published or not. The documents may come from teaching and research institutions in France or abroad, or from public or private research centers.
L'archive ouverte pluridisciplinaire HAL, est destinée au dépôt et à la diffusion de documents scientifiques de niveau recherche, publiés ou non, émanant des établissements d'enseignement et de recherche français ou étrangers, des laboratoires publics ou privés. 


\title{
Novel Perfluorinated Triblock Amphiphilic Copolymers for Lipid- Shelled Microbubble Stabilization
}

\author{
Yohann Corvis, ${ }^{*} \dagger$ Simona Manta, ${ }^{\dagger}$ Caroline Thebault, $^{\dagger}$ Olivier Couture, ${ }^{\ddagger}$ Hélène Dhotel, ${ }^{\dagger}$ \\ Jean-Philippe Michel, ${ }^{\S}, \|$ Johanne Seguin, ${ }^{\dagger}$ Michel Bessodes, ${ }^{\dagger}$ Philippe Espeau, ${ }^{\dagger}$ Chantal Pichon, ${ }^{\perp}$ \\ Cyrille Richard, $^{\dagger}$ and Nathalie Mignet $^{\dagger}$
}

†Team Vectors for Molecular Imaging and Targeted Therapy, Faculty of Pharmacy, Paris Descartes University, Sorbonne Paris Cité, CNRS UMR8258, INSERM U1022, Chimie ParisTech, PSL Research University, 4 avenue de l'Observatoire, 75006 Paris, France ${ }^{\ddagger}$ CNRS, INSERM, ESPCI ParisTech, Institut Langevin, PSL Research University, 75005 Paris, France

${ }^{\S}$ Univ Paris Sud, Institut Galien Paris Sud, Université Paris-Saclay, 5 rue Jean-Baptiste Clément, 92296 Châtenay-Malabry Cedex, France

"CNRS, UMR 8612, 92296 Châtenay-Malabry, France

${ }^{\perp}$ Centre de Biophysique Moléculaire, UPR 4301, Université d'Orléans, UFR Sciences, 45100 Orléans, France

\begin{abstract}
Amphiphilic triblock (Atri) copolymers made of perfluorinated alkyl chain linked to hydrocarbon chain and methoxypoly(ethylene glycol) of three different molecular weights were synthesized. In vitro evaluation demonstrated that these new compounds were noncytotoxic. Characterization and interaction of each triblock copolymer with a branched polyamine myristoyl lipid (2-\{3[bis-(3amino-propyl)-amino]-propylamino\}-N-ditetra-decyl carbamoyl methyl-acetamide, DMAPAP) were studied by the Langmuir film method and thermal analysis. The triblock copolymer/cationic lipids $(1: 10, \mathrm{w} / \mathrm{w})$ were mixed with perfluorobutane gas to form microbubbles (MBs). The latter were characterized by optical microscopy to get the microbubble size and concentration by densimetry to determine the amount of encapsulated gas and by ultrasound to assess oscillation properties. Atri with the lowest and intermediate weights were shown to interact with the cationic lipid DMAPAP and stabilize the Langmuir film. In that case, monodisperse microbubbles ranging from $2.3 \pm 0.1$ to $2.8 \pm 0.1 \mu \mathrm{m}$ were obtained. The proportion of encapsulated gas within the MB shell increased up to 3 times after the incorporation of the copolymer with the lowest and intermediate weights. Moreover, the acoustic response of the microbubbles was maintained in the presence of the copolymers.
\end{abstract}

\section{INTRODUCTION}

Contrast agents constituted of a lipid shell are currently used in clinical treatments to increase the signal-to-noise ratio of ultrasound imaging and improve diagnoses. ${ }^{1}$ However, these agents exhibit a poor stability with a half-life of $10 \mathrm{~min}$ for some commercially available microbubbles (MBs). Moreover, microbubbles have been used in combination with sonoporation to deliver drugs. Sonoporation can be used in to reach deep tissues without damage, in particular, using low ultrasound energy. Under ultrasound waves, microbubbles oscillate in all directions, leading to membrane destabilization, which has been described by different mechanisms: cavitation, mechanical streaming, and radiation forces. ${ }^{2}$ Therefore, association of microbubbles with sonoporation increases cell membrane permeability and thus performs a more efficient gene $^{3}$ or drug delivery. ${ }^{4}$

Recently, positively charged microbubbles have been developed in our laboratory to increase the amount of nucleic acid associated with the microbubbles. ${ }^{5}$ Even though the advantage of cationic microbubbles was only shown for low DNA doses and for local delivery, ${ }^{6}$ they may be interesting to transfect deep tissues, by increasing the amount of DNA reaching the tissue and improving the transfection level. ${ }^{7}$ Comparing neutral and cationic microbubbles, Wang et al. indeed showed a better inhibition of hind limb tumor growth with positively charged microbubbles. ${ }^{8}$ However, the levels of gene transfection reported remain rather low and the reproducibility of the transfection level obtained in vivo remains an issue. One of the reasons might be the low microbubble stability. If a stability below $1 \mathrm{~h}$ might be acceptable for ultrasound-enhanced imaging, stability should be increased for targeted ultrasound-enhanced contrast agents and for drug and gene delivery promoted by a combination of microbubbles and ultrasound. 


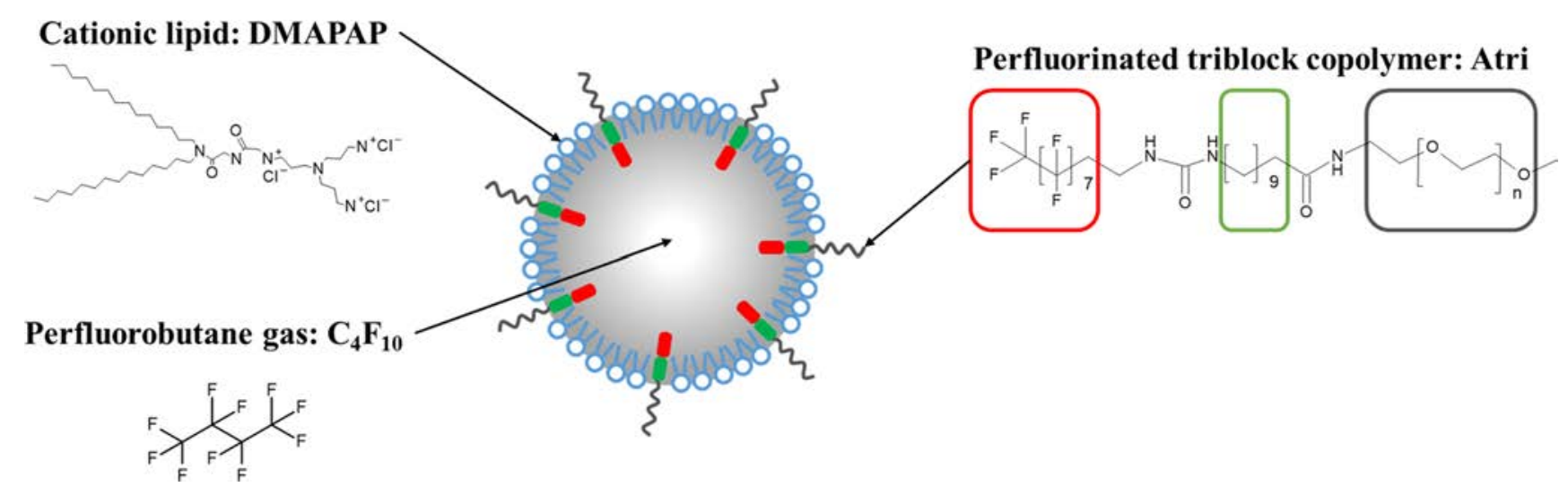

Figure 1. Schematic representation of microbubbles formed with cationic lipid 2-\{3[bis-(3-amino-propyl)-amino]-propylamino $\}$ - $N$-ditetradecyl carbamoyl methyl-acetamide (DMAPAP) (blue elements) mixed with amphiphilic triblock (Atri) copolymers (red, green, and dark gray assembly) and filled with perfluorobutane gas (light gray area). For the Atri copolymer representation, the red, green, and dark gray moieties represent the perfluorinated alkyl part, the hydrocarbon block, and the $a$-amino-e-methoxy-poly(ethylene glycol) (PEG) chain, respectively. $n=12,24$, and 44 for Atri500, Atri1000, and Atri2000, respectively.

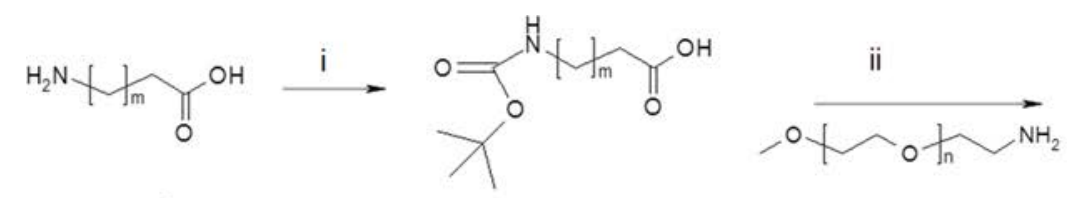

$m=9$

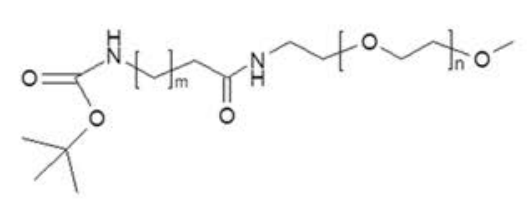

2: $n=11$

3: $\mathrm{n}=\mathbf{2 2}$

4: $n=44$

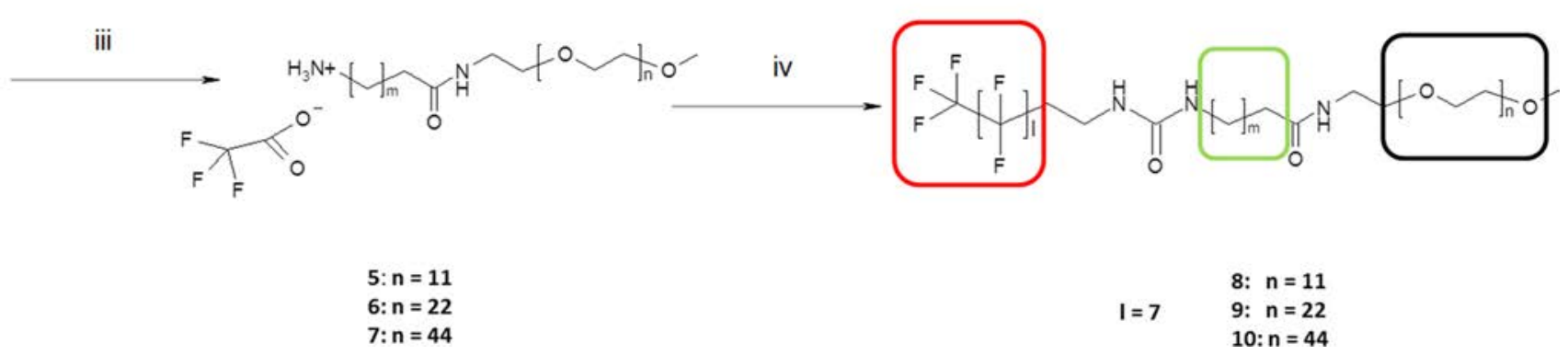

Figure 2. Synthetic procedure to prepare the three copolymers: Atri500 (8), Atri1000 (9), and Atri2000 (10). (i) $\mathrm{Boc}_{2} \mathrm{O}, \mathrm{Et}_{3} \mathrm{~N}$; (ii) $\mathrm{BOP} \mathrm{Et}_{3} \mathrm{~N}$; (iii) trifluoroacetic acid (TFA); (iv) heptadecafluorodecyl isocyanate, $\mathrm{Et}_{3} \mathrm{~N}$.

Two options have been proposed to increase microbubble stability: increasing the molecular weight of insoluble gas, which led to microbubbles filled with perfluorinated gas, or increasing the thickness of the microbubble shell. ${ }^{9,10}$ However, playing with the latter often inhibits the nonlinear oscillation of microbubbles and thus reduces their impact on membrane destabilization since the lipid shell is particularly suited for microbubble nonlinear oscillation. ${ }^{11}$ An alternative is to modulate the shell composition of the microbubbles. In this context, fluorinated amphiphiles have been proposed and shown to interact with phospholipids to form more stable organized self-assemblies. ${ }^{12,13}$

In designing the lipid shell and perfluorinated gas for this study, we took into consideration the data above and the ternary-phased microbubble specifications, i.e., the hydrophilic domain (outer side of the microbubble shell and water continuous medium), the hydrophobic domain (inner side of the microbubble shell), and the perfluorinated gas, to create triblock copolymers that would interact with the continuous medium.
The copolymer has been designed with the following: (i) a perfluorinated block to promote interaction with the gas core, (ii) a carbon alkyl chain to promote copolymer insertion into the microbubble membrane by hydrophobic interaction with the lipid shell, and (iii) a polyethylene chain both to stabilize the microbubbles toward the medium in which they will be dispersed and to prevent nonspecific interaction of microbubbles with biological components of the plasma (Figure 1).

In this work, the synthesis of three newly designed copolymers, i.e., Atri500, Atri1000, and Atri2000, is reported (cf. Supporting Information). We highlight their noncytotoxicity as well as their capacity to interact with the cationic lipid DMAPAP $^{14}$ to form microbubbles containing perfluorobutane gas, increasing its loading, and maintaining the nonlinear oscillation characteristics of the microbubbles.

\section{MATERIALS AND METHODS}

11-Aminoundecanoic acid, $\mathrm{Boc}_{2} \mathrm{O}$, (benzotriazol-1-yloxy)tris(dimethylamino)phosphonium hexafluorophosphate (BOP), and heptadecafluorodecyl isocyanate were purchased from Sigma-Aldrich. 
The solvents were obtained from SDS-Carlo Erba and aminomethoxy-poly(ethylene glycol)s from Iris Biotech.

DMAPAP Synthesis. Synthesis of $2-\{3$ [bis-(3-amino-propyl)amino]-propylamino $\}$ - $N$-ditetradecyl carbamoyl methyl-acetamide, otherwise referenced as DMAPAP, was reported by Thompson et al. ${ }^{14}$

Atri Synthesis. For the present study, 10 molecules were prepared in 4 steps starting from the commercial amino undecanoic acid, PEG, and perfluoroalkyl derivatives (Figure 2). The details of each synthesis, as well as the complete characterization of these 10 compounds by means of NMR and mass spectrometry (MS) analyses, are reported in the Supporting Information. Polydispersity of the compounds is the same as polydispersity of the PEGs from which they were synthetized (cf. Supporting Information).

${ }^{1} \mathrm{H},{ }^{13} \mathrm{C}$ NMR Spectroscopies, and Mass Spectrometry. ${ }^{1} \mathrm{H}$ and ${ }^{13} \mathrm{C}$ NMR spectra were recorded at 400 and $100 \mathrm{MHz}$, respectively. MS was performed on a LC-MS-2010 A device (Shimadzu, Japan) with UV $(220 \mathrm{~nm})$ and electrospray ionization detection and an Alltima HP $3 \mu$ column.

Cell Mitochondrial Activity Assessment (3-(4,5-Dimethylthiazol-2-yl)-2,5-diphenyltetrazolium Bromide, MTT). Murine BNL (BNL 1ME A.7R.1, ATCC TIB75) liver cells and murine $\mathrm{NIH} / 3 \mathrm{~T} 3$ (ATCC CRL1658) fibroblast cells were grown in Dulbecco's modified Eagle's medium containing $2 \mathrm{mM}$ L-glutamine, $10 \%$ fetal bovine serum (BNL 1ME A.7R.1) or bovine serum (NIH/ 3T3), $100 \mathrm{U} \mathrm{mL}^{-1}$ penicillin, and $100 \mathrm{mg} \mathrm{mL}^{-1}$ streptomycin $\left(37^{\circ} \mathrm{C}\right.$, $\left.5 \% \mathrm{CO}_{2}\right)$. The compounds were initially dissolved in water at a stock concentration of $20-40 \mathrm{mg} \mathrm{mL} \mathrm{m}^{-1}$ and were further diluted in cell culture medium. Cells in exponential growth were plated onto 96-well plates at 20000 cells per well in $100 \mu \mathrm{L}$ of culture medium. Twentyfour hours after plating, $100 \mu \mathrm{L}$ of medium containing DMAPAP or Atri were added on the cells and incubated for $24 \mathrm{~h}$ at $37^{\circ} \mathrm{C}$ and $5 \%$ $\mathrm{CO}_{2}$. After this exposure period, cell viability was assayed using the MTT test. This test, based on mitochondrial cell activity, gives insight into the intrinsic cytotoxicity of a given compound. ${ }^{15}$ The reactant, namely 3-(4,5-dimethylthiazol-2-yl)-2,5-diphenyltetrazolium bromide, is reduced by mitochondrial succinate dehydrogenase produced by viable cells. The reduction leads to formation of formazan, which is purple and insoluble in the medium. ${ }^{16,17}$ Hence, the initial medium, slightly yellow in color, turns to dark blue with an intensity proportional to the number of viable cells via their metabolic activity. The MTT reagent $\left(0.5 \mathrm{mg} \mathrm{mL}^{-1}\right.$ final concentration in the culture medium) was added in each well, and the plates were incubated overnight at $37^{\circ} \mathrm{C}$. After the incubation period, the supernatant was carefully removed and the formazan precipitate was solubilized in 100 $\mu \mathrm{L}$ of dimethyl sulfoxide. After $10-15 \mathrm{~min}$ agitation on an orbital shaker, the absorbance for $\lambda=562 \mathrm{~nm}$ was read in a microplate reader (Plate reader Infinite 200 PRO, Life Sciences, Tecan). Cell viability (\%, $n=4$ different and independent experiments) corresponds to the ratio of the values of the cells treated with the tested toxicity compound and the values of the control (ultrapure water, $0.2 \mu \mathrm{m}$ filtration).

Thermal Analysis. Differential scanning calorimetry (DSC) experiments were performed at $10 \mathrm{~K} \mathrm{~min}^{-1}$ from 5 to $60{ }^{\circ} \mathrm{C}$ under

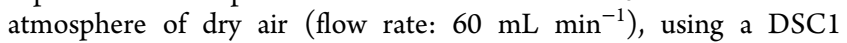
calorimeter (Mettler-Toledo, Switzerland), calibrated beforehand with high-purity indium $\left(99.99 \%, T_{\text {fus }}=156.6{ }^{\circ} \mathrm{C}\right.$, and $\Delta_{\text {fus }} H=$ $\left.28.45 \mathrm{~J} \mathrm{~g}^{-1}\right)$ and zinc $\left(99.99 \%, T_{\text {fus }}=419.6{ }^{\circ} \mathrm{C}\right.$, and $\Delta_{\text {fus }} H=107.5 \mathrm{~J}$ $\mathrm{g}^{-1}$ ) provided by Mettler-Toledo. The DSC samples were prepared by physical mixing, then weighed in aluminum pans using a microbalance sensitive to $1 \mu \mathrm{g}$ and hermetically sealed at room temperature. The experiments were performed three times to take into consideration the reproducibility of each preparation. The DSC curves are represented as the evolution of the heat flow normalized by the total mass of the sample $\left(\mathrm{W} \mathrm{g}^{-1}\right)$ vs the scanned temperature in Celsius degrees. The temperature data were determined at the intersection point between the tangent of the onset of the endothermic signal and the baseline. ${ }^{18,19}$ Enthalpies were recalculated as a function of the mass of the compound taken into consideration (cationic lipid or copolymer assembly). A DSC signal is obtained when the sample presents a transformation by absorbing (endother- mic transition) or releasing (exothermic transition) energy. When a given compound interacts with another one, one can notice a change of the DSC signal of the mixture by analyzing the temperature or enthalpy modification. The nature of the interaction can also be assessed.

Langmuir Monomolecular Film Technique. Monolayer compression isotherms were carried out using a thermostated KSV 2000 Langmuir balance (Biolin Scientific, Finland). Two hydrophilic Delrin barriers above a Teflon trough of $775.75 \mathrm{~cm}^{2}$ surface area were used for the symmetric compression of the monomolecular film at the air/water interface (compression rate: $5 \mathrm{~mm} \mathrm{~min}^{-1}$ barrier $^{-1}$ ). The device was equipped with a platinum Wilhelmy plate $(19.62 \times 10$ $\mathrm{mm}^{2}$ ) hooked to an electrobalance for obtaining the surface pressure (П) data. The device was caged in a plexiglass chamber to limit surface contamination, and temperature was kept constant at $20{ }^{\circ} \mathrm{C}$. Before each use, the trough and barriers were cleaned with chloroform and ethanol and then rinsed with pure water. All solvents used for cleaning the trough and the barriers were of analytical grade. Pure water (Millipore Direct $8,18.2 \mathrm{M} \Omega \mathrm{cm}$ ) of surface tension $(\gamma)$ of $72.75 \mathrm{mN} \mathrm{m}^{-1}$ at $20^{\circ} \mathrm{C}$ was used to prepare the subphase solutions in all experiments. Monolayers were spread on pure water using organic solutions of DMAPAP, Atri500, Atri1000, and Atri2000, as well as the DMAPAP/Atri $(10: 1 \mathrm{w} / \mathrm{w})$ mixtures, prepared with spectrophotometric grade chloroform (Aldrich, ACS) at a concentration $\sim 0.8 \mathrm{mg}$ $\mathrm{mL}^{-1}$. After $\sim 15 \mathrm{~min}$, allowing evaporation of the organic solvent, the films were compressed and the surface pressure-molecular area ( $\Pi-$ A) compression isotherm experiments were recorded. All compression isotherm experiments were repeated twice and were strictly reproducible. The squeeze out pressure corresponds to the pressure from which the molecule that interacts with the lipid monolayer is excluded from the latter into the subphase. The collapse point of the compression isotherm was determined at the breaking of the surface pressure for which a two-dimensional-three-dimensional transition occurs, indicating the end of stability of the monolayer. To better assess the nature of intermolecular interactions within the monolayers, the excess free energy of mixing $\left(\Delta G_{\text {excess }}\right)$ was calculated for each system using the method detailed by Goodrich, who demonstrated that one can have access to $\Delta G_{\text {excess }}$ from the compression isotherms of the single components and mixed film by integrating the $\Pi-A$ curves. ${ }^{20}$ For the present study, the integrations were calculated with respect to $\Pi$ between $0 \mathrm{mN} \mathrm{m}^{-1}$ and $\Pi_{\text {squeeze out }}$.

Microbubble Preparation. Four microbubble formulations were prepared with the cationic lipid in association with each triblock molecule: Atri500, Atri1000, and Atri2000, and one with the cationic lipid alone. A mixture of DMAPAP lipid and each Atri (10:1, w/w) was dissolved in chloroform, followed by solvent evaporation to form a thin film. Then, the film was hydrated with $5 \%$ sucrose solution to form micelles. Microbubbles were prepared by mechanical agitation, as previously described, ${ }^{5}$ with a final cationic lipid concentration of 1

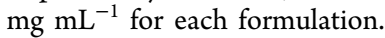

Microbubble Characterization by Optical Microscopy. The stability over time of each microbubble formulation was determined by optical microscopy using an Olympus BX 41TF microscope (Olympus Corporation, Tokyo, Japan) equipped with a camera. Microbubbles were diluted and placed on a $200 \mu \mathrm{m}$ Malassez hemocytometer cell for $2 \mathrm{~min}$ to allow all the bubbles to float on the top of the cell before five pictures were taken. Each measurement was repeated three times. The microbubble concentration and size were determined using particle counting in ImageJ software. ${ }^{5}$

Gas-Encapsulated Amount. The amount of perfluorobutane gas encapsulated into the microbubbles was determined by densimetry, before and after microbubble destruction ${ }^{21}$ with a DMA4500 densimeter (Anton Paar GmbH, Graz, Austria). For each formulation studied, freshly prepared microbubbles were first diluted in a $5 \%$ sucrose solution $(6 \times)$, then introduced into the oscillating U-tube, and their density was measured at $20^{\circ} \mathrm{C}$ ( $\left.\rho_{\text {microbubbles }}\right)$. Microbubbles were then burst in a water bath sonicator to eliminate the encapsulated perfluorobutane, and the density of the remaining solution was measured $\left(\rho_{\text {solution }}\right)$. Measures were repeated with eight different batches of the same formulation. The encapsulated 
perfluorobutane volume fraction percentage ${ }^{21}\left(V_{\text {gas }}\right)$ was determined as follows

$$
V_{\text {gas }}(\%)=100 \times\left(1-\frac{\rho_{\text {microbubbles }}}{\rho_{\text {solution }}}\right) \times 6
$$

Microbubble Acoustic Properties. To determine the microbubble acoustic response under ultrasound, a homemade setup was designed using a $1 \mathrm{MHz}$ Continuous Doppler probe as an ultrasound emission transducer (Blatek, State College PA, $10 \mathrm{~mm}$ diameter) connected to a 3Bop ultrasound generator (Image Guided Therapy, Pessac, France) and a $5 \mathrm{MHz}$ transducer for detection (Panametrics, Olympus, Center Valley, $13 \mathrm{~mm}$ diameter). Microbubbles or carboxylated polystyrene reference particles (CPC1000; IZON Science) were diluted 30 times in degassed water in a $4 \mathrm{~mL}$ cuvette placed between the two transducers, and measurements were repeated 5 times for each formulation. The acoustic response was monitored with a TiePie HS-5 oscilloscope (Tie-Pie, Sneek, Netherlands) and processed with Matlab (Mathworks, Natick) with the following ultrasound emission parameters: an acoustic pressure of $375 \mathrm{kPa}$, with a duty cycle of $10 \%$ and 20 pulses each of $100 \mu$ s.

\section{RESULTS AND DISCUSSION}

Synthesis. Amphiphilic triblock (Atri) copolymers are composed of a perfluorinated alkyl chain, a hydrocarbon chain, and a methoxy-poly(ethylene glycol) group of three different molecular weights $\left(500,1000\right.$, or $\left.2000 \mathrm{~g} \mathrm{~mol}^{-1}\right)$. They were obtained in four straightforward steps by successive condensations between the PEG and the alkyl chain, followed by the addition of perfluorinated alkyl chain (Figure 2 and the Supporting Information). The three methoxy PEG amines were independently reacted with Boc-amino-undecanoic acid in the presence of BOP and triethylamine to lead to the corresponding diblock alkyl-PEGs and were purified by precipitation in cold ether. Boc deprotection was obtained by treatment with trifluoroacetic acid (TFA) in dichloromethane to obtain the amines as TFA salt. Attempts to react the amines with different activated derivatives of perfluorinated acid either failed or led to incomplete reactions. BOP condensation, leading to the intermediate benzotriazole activated ester, the anhydride generated by dicyclohexylcarbodiimide reaction, and the acid chloride obtained by thionyl chloride-mediated activation, were unsuccessful. From these data, it has been postulated that the presence of fluorine induced high reactivity of these derivatives and made them highly susceptible to hydrolysis by traces of water that are almost unavoidably present in the PEG molecule. Then, heptadecafluorodecyl isocyanate has been chosen since it led quantitatively to the corresponding urea condensation products.

Evaluation of the DMAPAP, Atri500, and Atri1000 Cytotoxicity. As presented in Figure 3, the Atri500 and Atri1000 copolymers show low cytotoxicity on NIH/3T3 cells or on BNL cells after $24 \mathrm{~h}$ of exposure. For DMAPAP, a half maximal inhibitory concentration $\left(\mathrm{IC}_{50}\right)$ of $69 \pm 10$ and $40 \pm$ $3 \mu \mathrm{g} \mathrm{mL}^{-1}$ was obtained for $\mathrm{NIH} / 3 \mathrm{~T} 3$ and BNL cell lines, respectively. Since the final concentration of DMAPAP for the chosen formulations are comprised between 30 and $100 \mu \mathrm{g}$ $\mathrm{mL}^{-1}$ and that of Atri500 and Atri1000 are 10 times lower, one can consider DMAPAP and the copolymer with the lowest and intermediate weights as biocompatible compounds.

Characterization of Copolymers/Lipid Interaction Using Differential Scanning Calorimetry. As far as endothermic behavior is concerned, calorimetry experiments performed with DMAPAP samples indicate an endothermic lipid main transition at $24.8 \pm 0.1^{\circ} \mathrm{C}$ with an associated energy
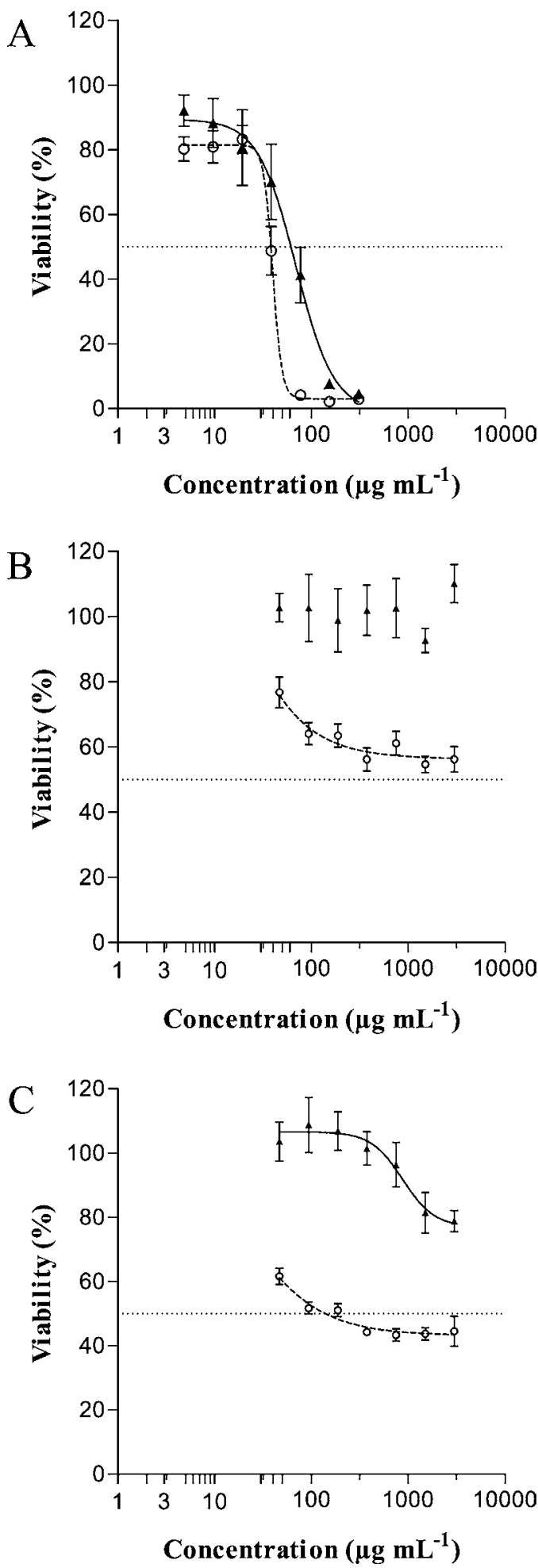

Figure 3. NIH/3T3 ( $\boldsymbol{\Delta}$, solid line) and $\mathrm{BNL}(\mathrm{O}$, dotted line) cell lines viability after $24 \mathrm{~h}$ exposure of DMAPAP (A), Atri500 (B), and Atri1000 (C) measured by MTT assay $(n=4)$. The curve profile was obtained using the sigmoidal dose-response (variable slope) equation (Graph pad prism software). The dotted horizontal line indicates $50 \%$ of viability.

$\Delta_{\text {trans }} H=23.7 \pm 0.1 \mathrm{~J} \mathrm{~g}^{-1}$ (Figure $4 \mathrm{~A}-\mathrm{C}$, middle curves). In analogy with the 1,2-dimyristoyl-sn-glycero-3-phosphocholine (DMPC) main transition signal, the DMAPAP endotherm corresponds to a gel $\rightarrow$ liquid crystalline phase. ${ }^{22,23}$ The three 

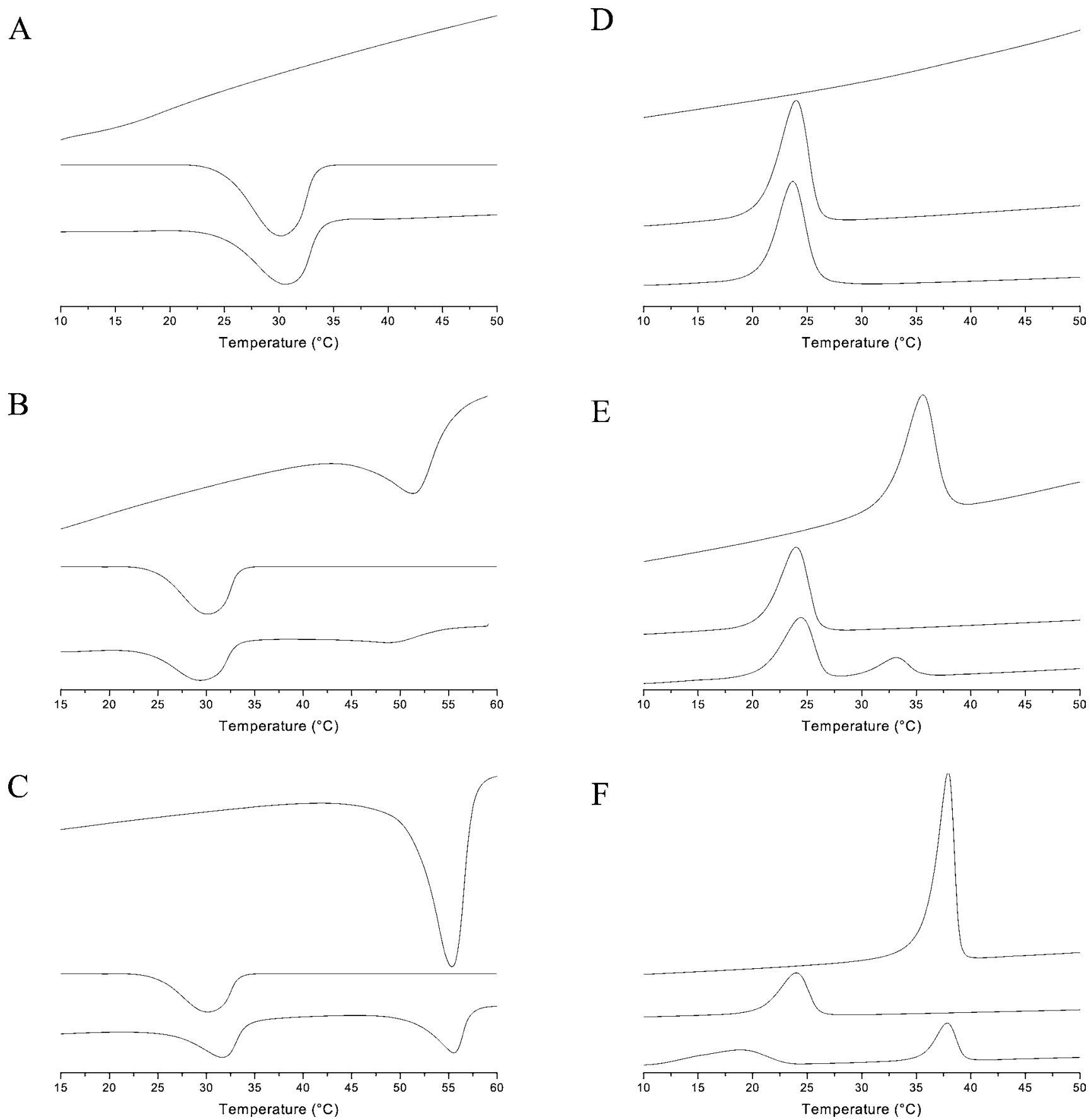

Figure 4. DSC curves obtained for copolymer (upper curve), DMAPAP (middle curve), and the corresponding 10:1 physical mixtures (lower curve). The results obtained when copolymer is Atri500, Atri1000, and Atri2000 are gathered in panels (A, D), (B, E), and (C, F), respectively. Signals were recorded upon heating $(A-C)$ and upon cooling $(D, E)$. Ordinate: mass-normalized heat flow in $\mathrm{W} \mathrm{g}^{-1}$. The curves were shifted for clarity. Exothermic signals are pointing up.

copolymers tested present a singular thermal behavior with high-capacity heat and transition signals at relatively high temperature for Atri1000 and Atri2000. No transition has been observed with Atri500. All phase transition temperatures and enthalpies determined from the DSC curves recorded upon heating and upon cooling are gathered in Table 1.

DMAPAP transition temperature is not modified in presence of Atri500 $\left(T_{\text {trans }}=24.7 \pm 0.1{ }^{\circ} \mathrm{C}\right)$, indicating a potential noninvasive behavior of the Atri copolymer in presence of DMAPAP. This hypothesis is confirmed by the invariant heat of transition $\left(\Delta_{\text {trans }} H=23.6 \pm 0.5\right.$ and $24.6 \pm 0.5 \mathrm{~J} \mathrm{~g}^{-1}$ for
DMAPAP and the solid physical mixture). As far as the copolymer Atri1000 is concerned, one can notice a slight decrease of the transition temperature of DMAPAP in presence of the copolymer, i.e., $24.8-23.7{ }^{\circ} \mathrm{C}$, but also for the Atri1000 signal, indication of potential lipid/copolymer intermolecular interactions (e.g., ion-induced dipole forces, hydrogen bonds, and van der Waals forces). However, the corresponding heat is not significantly modified, nor is the energy of the Atri1000 transition. Interestingly, when Atri2000 is mixed with DMAPAP, the transition temperature increases by $1.4{ }^{\circ} \mathrm{C}\left(26.2 \pm 0.1{ }^{\circ} \mathrm{C}\right)$ and the copolymer heat of transition 
Table 1. Phase Transition Temperatures and Enthalpies for the Cationic Lipid (DMAPAP), the Copolymers (Atri), and Their 10:1 Solid Physical Mixtures Measured Upon Heating and Cooling of the Sample

\begin{tabular}{|c|c|c|c|c|c|}
\hline & \multirow[b]{2}{*}{ sample } & \multicolumn{2}{|c|}{ heating } & \multicolumn{2}{|c|}{ cooling } \\
\hline & & $T_{\text {trans }}\left({ }^{\circ} \mathrm{C}\right) \pm 0.1-0.2$ & $\Delta_{\text {trans }} H\left(\mathrm{~J} \mathrm{~g}^{-1}\right) \pm 0.5-0.9$ & $T_{\text {trans }}\left({ }^{\circ} \mathrm{C}\right) \pm 0.1-0.2$ & $\Delta_{\text {trans }} H\left(\mathrm{~J} \mathrm{~g}^{-1}\right) \pm 0.5-0.9$ \\
\hline \multirow[t]{4}{*}{ lipid signal } & DMAPAP & 24.8 & 23.6 & 26.0 & -29.7 \\
\hline & DMAPAP/Atri500 & 24.7 & 24.6 & 25.9 & -28.5 \\
\hline & DMAPAP/Atri1000 & 23.7 & 24.4 & 26.6 & -28.1 \\
\hline & DMAPAP/Atri2000 & 26.2 & 24.8 & 22.9 & -28.6 \\
\hline \multirow[t]{6}{*}{ copolymer signal } & Atri500 & & & & \\
\hline & Atri1000 & 45.8 & 31.8 & 37.8 & -47.0 \\
\hline & Atri2000 & 51.1 & 90.6 & 39.0 & -90.9 \\
\hline & DMAPAP/Atri500 & & & & \\
\hline & DMAPAP/Atri1000 & 42.1 & 31.9 & 35.3 & -33.9 \\
\hline & DMAPAP/Atri2000 & 51.2 & 77.4 & 39.4 & -80.1 \\
\hline
\end{tabular}

drastically decreases $\left(90.6-77.4 \mathrm{~J} \mathrm{~g}^{-1}\right)$, suggesting unfavorable lipid/copolymer intermolecular interactions. These interpretations are also confirmed by the results obtained during cooling (Figure 4D-F), indicating the reversible DMAPAP and Atri phase transitions within the physical mixtures.

The latter result can inform us about a possible interaction between the lipid and the copolymer. To gain insight into the interactions between them at a hydrophilic/hydrophobic interface mimicking the microbubble interface, interfacial behavior of the lipid, copolymers, and their mixtures has been investigated by means of the Langmuir monomolecular film approach.

Evaluation of Copolymers/Lipid Interaction at the Hydrophilic/Hydrophobic Interface Using the Langmuir Monomolecular Film Approach. The resulting compression isotherms are presented in Figure 5. DMAPAP presents a monomolecular film behavior at the water/air interface, as expected for an amphiphilic compound. With a take-off of the surface pressure at $125 \AA^{2}$ molecule $^{-1}$, a liquid condensed monomolecular film state, and a collapse at $\sim 54 \AA^{2}$ molecule ${ }^{-1}$ (Figure 5A), DMAPAP interfacial behavior is more or less similar to that of dimyristoyl common compounds such as dimyristoylphosphatidic acid ${ }^{24}$ or DMPC. ${ }^{25}$ As far as the interfacial behavior of each Atri copolymer is concerned (Figure 5B), the compression isotherms we obtained highlight three interesting features: (i) Atri copolymers form monomolecular films due to their relatively high amphiphilic properties, (ii) they present a nonideal gaseous phase at high molecular areas, and a liquid condensed-liquid expanded phase transition around $100 \AA^{2}$ molecule ${ }^{-1}$, and (iii) Atri2000 molecules seem to self-agglomerate at the hydrophilic/ hydrophobic interface.

DMAPAP/Atri compression isotherms first indicate that the cationic lipid and the copolymer interact to form a mixed monomolecular film for the DMAPAP/Atri500 and the DMAPAP/Atri1000 samples (Figure 5C). Here, the interactions are favored by specific design of the copolymer driven by hydrophobic interactions between the Atri hydrocarbon and perfluorinated blocks and the DMAPAP aliphatic chain on one hand, and by hydrophilic interactions between the Atri PEG moieties and the DMAPAP polar head on the other hand. Interestingly, for these two cases, the overall shape of the isotherm of the mixed film is not drastically different from that of the isotherm of DMAPAP alone or even from that of the given copolymer, demonstrating that the DMAPAP/Atri interactions are noninvasive. The shift of the isotherms obtained for the DMAPAP/Atri500 and DMAPAP/Atri1000 mixtures toward higher molecular areas compared with DMAPAP isotherm proves that Atri500 and Atri1000 interact with the cationic lipid even at the most condensed state of the film, i.e., a surface pressure of around $31 \mathrm{mN} \mathrm{m}^{-1}$ (cf. squeeze out pressures in Table 2). This value corresponds to the common surface pressure of biological membranes. ${ }^{26}$ For higher surface pressures, the Atri molecules are squeezed out of the DMAPAP monolayer, since the isotherms overlap. This phenomenon has frequently been observed for chemical compounds such as perfluoroalkyl chains ${ }^{27}$ or drugs ${ }^{28}$ interacting with phospholipids at a hydrophilic/hydrophobic interface. However, in the present study, it is noteworthy that the pressure at collapse, i.e., after squeezing out of the copolymer, is higher for the mixtures than that of pure DMAPAP (Figure 5C). This result confirms the interactions established between the polar head of the cationic lipid forming the monolayer and the pegylated part of the copolymer. Nevertheless, when the DMAPAP is mixed with Atri2000, the latter compound induces aggregation of the lipid layer at the interface ${ }^{29-31}$ since mean molecular DMAPAP area for the mixture after the liquid expanded-liquid condensed phase transition is lower than that obtained for pure DMAPAP monolayer. Indeed, the DMAPAP/Atri2000 isotherm should at least overlap the DMAPAP isotherm to justify a monolayer behavior.

The qualitative interpretation of the compression isotherms presented so far can also be confirmed by some interfacial parameters that are determined from the isotherms. The corresponding data give insights into the physicochemical properties of the layer studied as a function of the surface pressure of the interfacial film. From such data gathered in Table 2 for DMAPAP and the mixed DMAPAP/Atri films, one can confirm that Atri500, Atri1000, and Atri2000 interact with DMAPAP at a hydrophilic/hydrophobic interface. Excess Gibbs energies of mixing ${ }^{20,32}\left(\Delta G_{\text {excess }}\right)$ are negative for the three systems, indicating attractive interactions between the lipid and the copolymer and higher thermodynamic stability of the mixed monolayer (i.e., with Atri500 or Atri1000) compared to that of the pure monolayer. ${ }^{30,33}$ Indeed, when DMAPAP is mixed with either Atri500 or Atri1000, the interfacial behavior of the cationic lipid monolayer is not disturbed by the interaction with the copolymer, even after squeeze out of the latter $\left(\Pi_{\text {collapse }}\right.$ higher in the presence of the copolymers). Furthermore, the rigidity of the mixed monomolecular film in its most condensed state is higher in the presence of Atri500 or Atri1000 compared to that of the pure DMAPAP monolayer in the same state. This result indicates 

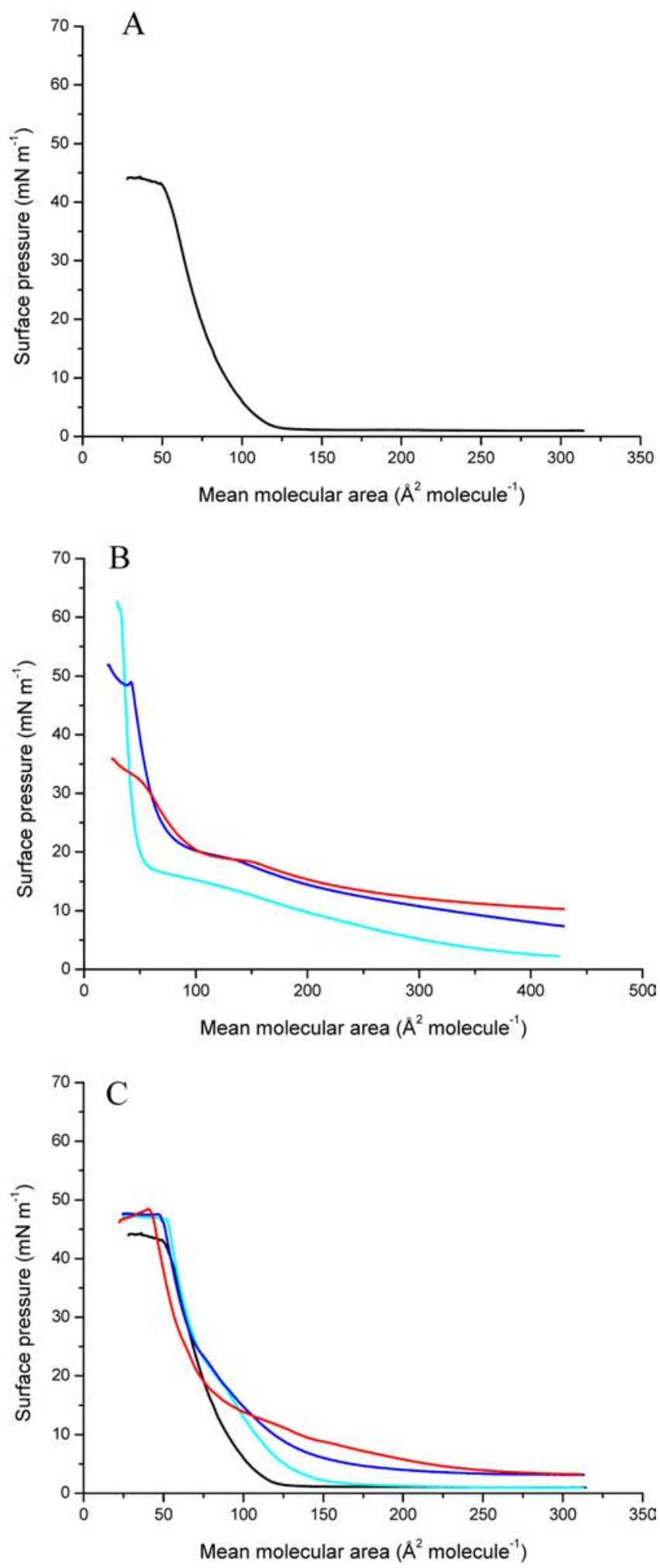

Figure 5. Compression isotherms obtained at the air/water interface for DMAPAP (A), Atri compounds (B), and 10:1 DMAPAP/Atri mixtures (C). Curve color code: pure DMAPAP (black), Atri500 or DMAPAP/Atri500 (cyan), Atri1000 or DMAPAP/Atri1000 (blue), and Atri2000 or DMAPAP/Atri2000 (red).

that the DMAPAP layer is stabilized when it is mixed with Atri500 or Atri1000. On the other hand, in the presence of Atri2000, the DMAPAP layer is more fluid. This results from the tendency of Atri2000 to aggregate the cationic lipid. ${ }^{30,31}$
Table 2. Interfacial Parameters for the DMAPAP (D) and the 10:1 DMAPAP/Atri Mixtures with Atri500 $\left(A_{1}\right)$, Atri1000 $\left(A_{2}\right)$, and Atri2000 $\left(A_{3}\right)$

$$
\begin{aligned}
& A_{\text {squeeze out }}\left(\AA^{2} \text { molecule }^{-1}\right) \\
& \Pi_{\text {squeeze out }}\left(\mathrm{mN} \mathrm{m}^{-1}\right) \\
& A_{\text {collapse }}\left(\AA^{2} \text { molecule }^{-1}\right) \\
& \Pi_{\text {collapse }}\left(\mathrm{mN} \mathrm{m}^{-1}\right) \\
& A_{0}\left(\AA^{2} \text { molecule }^{-1}\right) \\
& \Delta G_{\text {excess }}\left(\mathrm{J} \mathrm{mol}^{-1}\right) \\
& C_{\mathrm{s}}^{-1}\left(\mathrm{mN} \mathrm{m}^{-1}\right)
\end{aligned}
$$

\begin{tabular}{crrr}
\multicolumn{4}{c}{ samples } \\
\hline$D$ & $D / A_{1}$ & $D / A_{2}$ & $D / A_{3}$ \\
& 63.3 & 62.0 & 54.9 \\
& 31.8 & 31.1 & 31.9 \\
54.2 & 52.9 & 50.0 & 43.6 \\
40.4 & 46.2 & 46.1 & 46.8 \\
92.8 & 83.4 & 81.6 & 75.9 \\
& -85.8 & -435.8 & -485.7 \\
56.7 & 81.3 & 69.4 & 54.1
\end{tabular}

The latter interpretation is consistent with the fact that the mean molecular area at the film collapse is $20 \%$ lower in presence of Atri2000 compared with pure DMAPAP.

All calorimetric and interfacial data converge toward the fact that nondenaturing interactions can be established between DMAPAP and Atri500 or Atri1000, stabilizing the monolayer. This is not the case with Atri2000. Such results validate the choice of DMAPAP/Atri500 or DMAPAP/Atri1000 lipid shells to stabilize gas microbubbles dispersed in aqueous solutions.

Microbubble Characterization by Optical Microscopy. Monodisperse microbubbles were obtained with the cationic lipid combined with Atri500 and Atri1000. Their mean size was unchanged in comparison with the microbubbles formed with the cationic lipid alone $(2.5 \pm 0.3 \mu \mathrm{m})$. The microbubbles obtained with Atri2000 had a mean size of $3.5 \pm 1.2 \mu \mathrm{m}$ and a large size distribution (Figure 6). This is
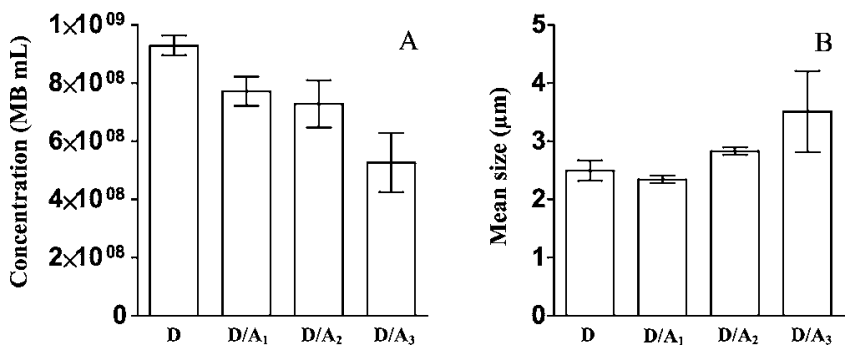

Figure 6. Mean microbubble concentration (A) and mean size (B) obtained with formulations of DMAPAP lipid alone $(D)$ and for DMAPAP with Atri500 $\left(D / A_{1}\right)$, Atri1000 $\left(D / A_{2}\right)$, and Atri2000 (D/ $\left.A_{3}\right)$. Values are means \pm standard error of the mean (SEM), $n=3$.

probably due to the formation of foam in presence of longer poly(ethylene glycol) chain. This hypothesis is confirmed by the number of $\mathrm{MB}$ by milliliters of solution, i.e., $(9.3 \pm 0.6) \times$ $10^{8}$ for DMAPAP microbubbles, $(7.7 \pm 0.9) \times 10^{8}$ for DMAPAP/Atri500, $(7.3 \pm 1.4) \times 10^{8}$ for DMAPAP/Atri1000, and $(5.3 \pm 1.8) \times 10^{8}$ for DMAPAP/Atri2000.

Stability studies were performed and showed that Atri500 and Atri1000 tend to stabilize the microbubbles over time, whereas Atri2000 destabilizes them (Figure 7). This is consistent with the destabilization observed with Langmuir experiments.

Apart from stability enhancement, one can wonder whether the presence of the perfluorinated copolymer in the formulation increases or not the amount of gas that could be encapsulated. The amount of encapsulated gas is usually not given for commercial microbubble formulations. However, the present study may allow discrimination between the different 


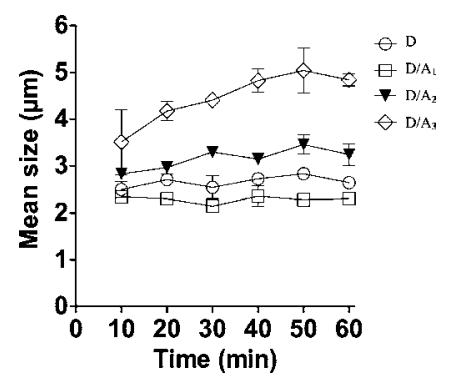

Figure 7. Size evolution over time of microbubbles obtained with formulations of DMAPAP lipid alone $(D)$ and for DMAPAP with Atri500 $\left(D / A_{1}\right)$, Atri1000 $\left(D / A_{2}\right)$, and Atri2000 $\left(D / A_{3}\right)$ (values are means \pm SEM, $n=3)$.

microbubble formulations prepared. Performing densimetry experiments, the amount of gas has been quantified in each formulation composed of the cationic lipid alone or the cationic lipid and Atri500 or Atri1000 mixtures. Data presented in Figure 8 shows that results were significantly

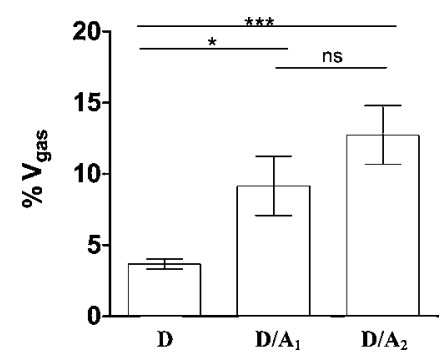

Figure 8. Encapsulated perfluorobutane volume fraction measured for microbubbles of DMAPAP lipid alone $(D)$ and for DMAPAP with Atri500 $\left(D / A_{1}\right)$ and Atri1000 $\left(D / A_{2}\right)$. Values are means \pm SEM, $n=$ 8.
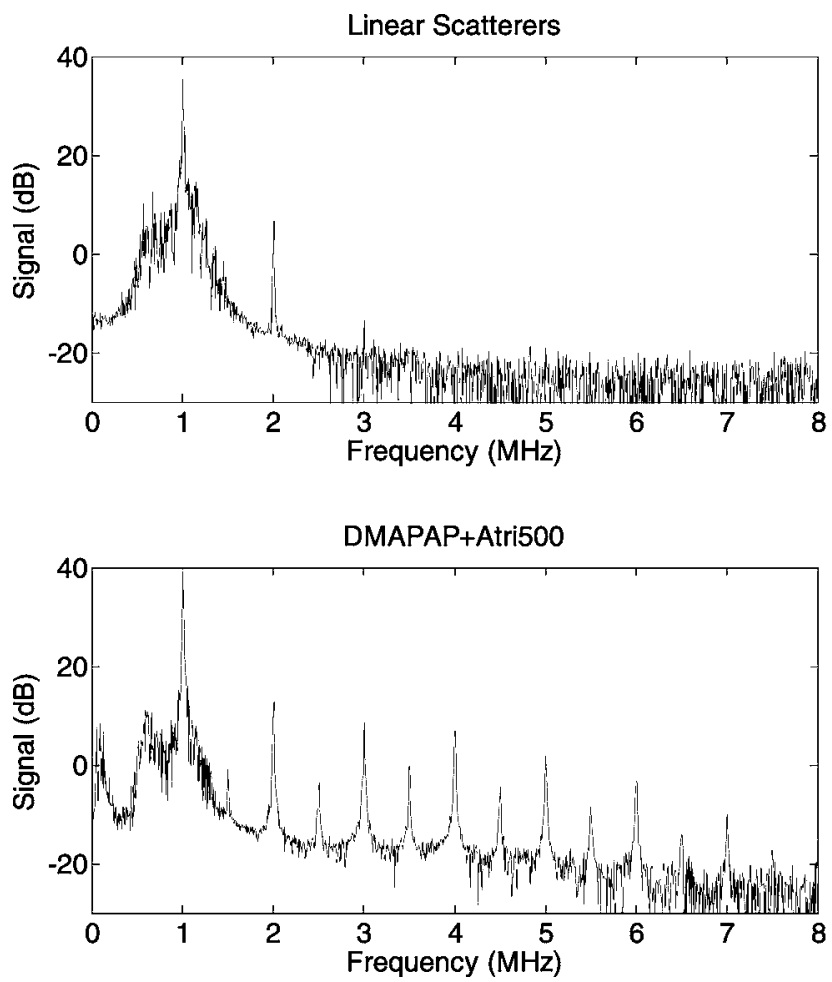

different between the three groups. The percentage of gas volume fraction was increased by a factor 2 with the addition of Atri500 and by a factor 3 with the addition of Atri1000. As the size of the microbubbles was unchanged despite the presence of the copolymers, a higher volume of gas means that the gas was more condensed within the microbubbles. This result can only be explained by the interaction between the perfluorinated part of the Atri copolymer and the encapsulated perfluorinated gas. It is noteworthy that the DMAPAP + Atri2000 system gives inconsistent results when studied by densimetry. This can be explained by the fact that the latter system is nonstable and heterogeneous due to the important size of the microbubbles (Figure 7).

Finally, as it has been demonstrated that Atri copolymers could be incorporated in the microbubble lipid layer, one can wonder whether the resulting increased stability could impair the acoustic properties of the new microbubble formulations. Nonlinear oscillation is indeed dependent on the deformation of the lipid shell. Using a homemade detection setup, the acoustic response was compared after ultrasound emission at 1 $\mathrm{MHz}$ by monitoring the echo from DMAPAP, DMAPAP/ Atri500, and DMAPAP/Atri1000 microbubbles and reference particles (polystyrene). The power spectrum of all MB types exhibited harmonics (multiples of the fundamental frequency) and subharmonics (half of multiples of the fundamental frequency), showing a nonlinear oscillation, whereas the reference particles exhibit a linear response (Figure 9). Similar intensities of harmonics and subharmonics were obtained for the three microbubble formulations. This indicates that the addition of Atri500 or Atri1000 does not inhibit the nonlinear oscillation response of cationic microbubbles.
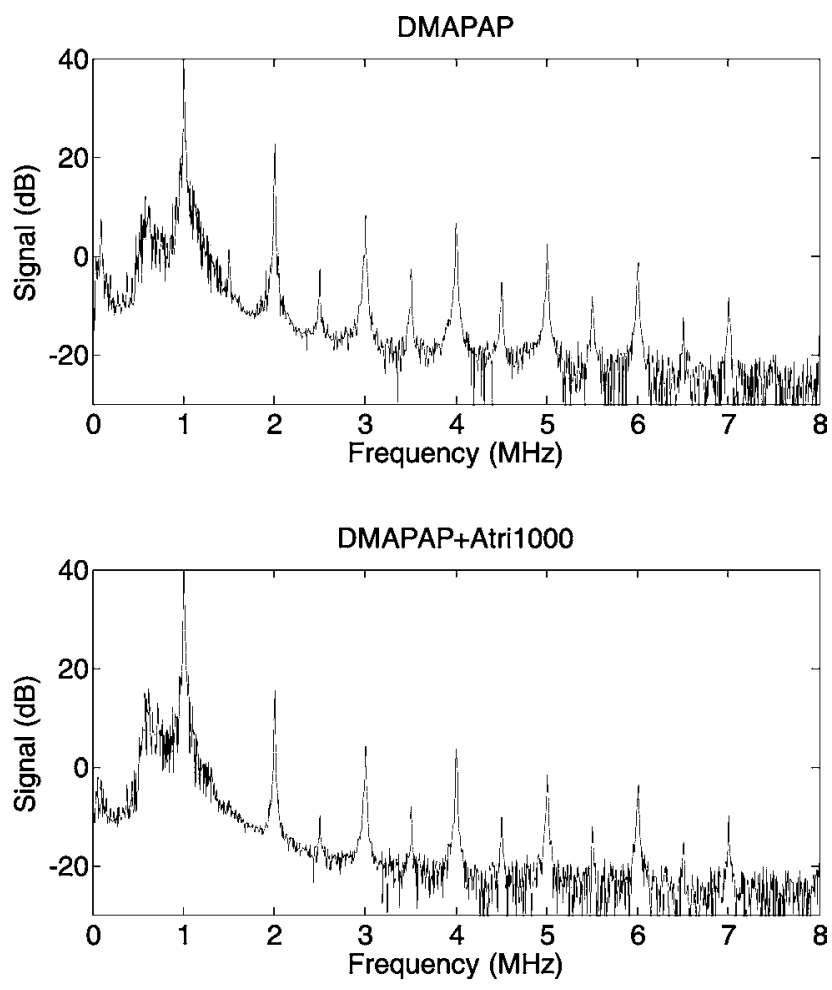

Figure 9. Power spectrum of linear scatterers, DMAPAP alone, DMAPAP/Atri500, and DMAPAP/Atri1000 microbubbles. 


\section{CONCLUSIONS}

This study shows that a polyamine myristoyl lipid, when mixed with copolymer amphiphiles, can be used to encapsulate perfluorobutane gas with efficient stability and improvement of the encapsulation rate. Nondestructive interactions established between the copolymer and the lipid allow for the formation of a more rigid lipid shell. Under these circumstances, the encapsulated gas is more condensed than in the system without copolymer. Moreover, the length of the copolymer PEG moieties influences the stability of the lipid shell. Hydrophilic/hydrophobic interfacial experiments showed that copolymers containing 44 moieties cause aggregation of cationic lipids and subsequent destabilization of the lipid shell monolayer. By contrast, copolymers containing 12 or 24 moieties increase stability of the microbubble shell due to nondestructive lipid/copolymer interactions. DMAPAP/ Atri500 and DMAPAP/Atri1000 were thus proved to be the best candidate systems to improve the percentage of encapsulated gas compared to pure DMAPAP. Since the quantity of therapeutic gas delivered with different microbubble formulations is usually not well established, the physicochemical ex- and in vitro studies performed here provide novel insights into the relative stability and biocompatibility, the gas encapsulation rate, and the response to ultrasound stimulation of microbubble systems. As microbubble stability is a critical selection criterion for pharmaceutical applications, this study will have wide applications for the development of contrast agents for medical imaging and bioavailability of therapeutic agents after sonoporation.

\section{ASSOCIATED CONTENT}

\section{Supporting Information}

The Supporting Information is available free of charge on the ACS Publications website at DOI: 10.1021/acs.langmuir.8b01668.

Atri synthesis; ${ }^{1} \mathrm{H},{ }^{13} \mathrm{C} \mathrm{NMR}$, and mass spectra for compounds $\mathbf{1}, \mathbf{5}, \mathbf{6}$, and 7 (PDF)

\section{AUTHOR INFORMATION}

\section{Corresponding Author}

E-mail: yohann.corvis@parisdescartes.fr.

\section{ORCID ${ }^{\circ}$}

Yohann Corvis: 0000-0002-4865-3348

Cyrille Richard: 0000-0003-4896-6675

\section{Notes}

The authors declare no competing financial interest.

\section{ACKNOWLEDGMENTS}

For fruitful discussions and advice on the manuscript, the authors gratefully acknowledge Karine Debbasch and Ruth Yoo. This work has been funded by the French National Research Agency (ANR MEDDU).

\section{REFERENCES}

(1) Richard, C.; Doan, B.-T.; Mignet, N. Increased Sensitivity for Medical Imaging Using Non-Ionizing Nanomedicine as Contrast Agents. In Nanotheranostics for Personalized Medicine; Mura, S., Couvreur, P., Eds.; World Scientific Publishing: Singapore, 2016; Chapter 2, pp 7-44.

(2) Delalande, A.; Kotopoulis, S.; Postema, M.; Midoux, P.; Pichon, C. Sonoporation: Mechanistic insights and ongoing challenges for gene transfer. Gene 2013, 525, 191-199.
(3) Sirsi, S. R.; Borden, M. A. Advances in ultrasound mediated gene therapy using microbubble contrast agents. Theranostics 2012, 2, $1208-1222$.

(4) Rizzitelli, S.; Giustetto, P.; Faletto, D.; Delli Castelli, D.; Aime, S.; Terreno, E. J. Controlled Release 2016, 230, 57-63.

(5) Manta, S.; Bessodes, M.; Bureau, M.-F.; Scherman, D.; Delalande, A.; Pichon, C.; Mignet, N. Characterization of Positively Charged Lipid Shell Microbubbles with Tunable Resistive Pulse Sensing (TRPS) Method: A Technical Note. Ultrasound Med. Biol. 2016, 42, 624-630.

(6) Panje, C. M.; Wang, D. S.; Pysz, M. A.; Paulmurugan, R.; Ren, Y.; Tranquart, F.; Tian, L.; Willmann, J. K. Ultrasound-mediated gene delivery with cationic versus neutral microbubbles: effect of DNA and microbubble dose on in vivo transfection efficiency. Theranostics 2012, 2, 1078-1091.

(7) Manta, S.; Renault, G.; Delalande, A.; Couture, O.; Lagoutte, I.; Seguin, J.; Lager, F.; Houze, P.; Midoux, P.; Bessodes, M.; Scherman, D.; Bureau, M.-F.; Marie, C.; Pichon, C.; Mignet, N. Cationic microbubbles and antibiotic-free miniplasmid for sustained ultrasound-mediated transgene expression in liver. J. Controlled Release 2017, 262, 170-181.

(8) Wang, D. S.; Panje, C.; Pysz, M. A.; Paulmurugan, R.; Rosenberg, J.; Gambhir, S. S.; Schneider, M.; Willmann, J. K. Cationic versus neutral microbubbles for ultrasound-mediated gene delivery in cancer. Radiology 2012, 264, 721-732.

(9) Dayton, P. A.; Zhao, S.; Bloch, S. H.; Schumann, P.; Penrose, K.; Matsunaga, T. O.; Zutshi, R.; Doinikov, A.; Ferrara, K. W. Application of ultrasound to selectively localize nanodroplets for targeted imaging and therapy. Mol. Imaging 2006, 5, 160-174.

(10) Maldiney, T.; Mignet, N.; Cornier, J.; Owen, A.; Kwade, A.; Van de Voorde, M. Pharmaceutical Nanotechnology: Innovation and Production; Wiley-VCH Verlag GmbH \& Co. KGaA: Weinheim, Germany, 2016; pp 533-560.

(11) Qin, S.; Caskey, C. F.; Ferrara, K. W. Phys. Med. Biol. 2009, 54, R27-R57.

(12) Hiranita, T.; Nakamura, S.; Kawachi, M.; Courrier, H. M.; Vandamme, T. F.; Krafft, M.-P.; Shibata, O. Miscibility behavior of dipalmitoylphosphatidylcholine with a single-chain partially fluorinated amphiphile in Langmuir monolayers. J. Colloid Interface Sci. 2003, 265, 83-92.

(13) Hoda, K.; Nakahara, H.; Nakamura, S.; Nagadome, S.; Sugihara, G.; Yoshino, N.; Shibata, O. Langmuir monolayer properties of the fluorinated-hydrogenated hybrid amphiphiles with dipalmitoylphosphatidylcholine (DPPC). Colloids Surf., B 2006, 47, 165-175.

(14) Thompson, B.; Mignet, N.; Hofland, H.; Lamons, D.; Seguin, J.; Nicolazzi, C.; de la Figuera, N.; Kuen, R. L.; Meng, X. Y.; Scherman, D.; Bessodes, M. Neutral postgrafted colloidal particles for gene delivery. Bioconjugate Chem. 2005, 16, 608-614.

(15) Hamid, R.; Rotshteyn, Y.; Rabadi, L.; Parikh, R.; Bullock, P. Comparison of Alamar Blue and MTT assays for high through-put screening. Toxicol. In Vitro 2004, 18, 703-710.

(16) Fotakis, G.; Timbrell, J. A. In vitro cytotoxicity assays: comparison of $\mathrm{LDH}$, neutral red, MTT and protein assay in hepatoma cell lines following exposure to cadmium chloride. Toxicol. Lett. 2006, 160, 171-177.

(17) Zeng, N.; Mignet, N.; Dumortier, G.; Olivier, E.; Seguin, J.; Maury, M.; Scherman, D.; Rat, P.; Boudy, V. Poloxamer bioadhesive hydrogel for buccal drug delivery: Cytotoxicity and trans-epithelial permeability evaluations using TR146 human buccal epithelial cell line. Int. J. Pharm. 2015, 495, 1028-1037.

(18) Mansour, H.; Wang, D.-S.; Chen, C.-S.; Zografi, G. Comparison of Bilayer and Monolayer Properties of Phospholipid Systems Containing dipalmitoylphosphatidylglycerol and dipalmitoylphosphatidylinositol. Langmuir 2001, 17, 6622-6632.

(19) Arora, M.; Bummer, P. M.; Lehmler, H.-J. Interaction of a partially fluorinated heptadecanoic acid with diacyl phosphatidylcholines of varying chain length. Langmuir 2003, 19, 8843-8851. 
(20) Goodrich, F. C. Molecular Interaction in Mixed Monolayers. In Proceedings of the International Congress Surface Activity; Butterworths: London, 1957; pp 85-91.

(21) Bjerknes, K.; Sontum, P. C.; Smistad, G.; Agerkvist, I. Preparation of polymeric microbubbles: formulation studies and product characterization. Int. J. Pharm. 1997, 158, 129-136.

(22) Ali, S.; Minchey, S.; Janoff, A.; Mayhew, E. A differential scanning calorimetry study of phosphocholines mixed with paclitaxel and its bromoacylated taxanes. Biophys. J. 2000, 78, 246-256.

(23) Almeida, C.; Lamazière, A.; Filleau, A.; Corvis, Y.; Espeau, P.; Ayala-Sanmartin, J. Membrane re-arrangements and rippled phase stabilization by the cell penetrating peptide penetratin. Biochim. Biophys. Acta, Biomembr. 2016, 1858, 2584-2591.

(24) Caseli, L.; Perinotto, A. C.; Viitala, T.; Zucolotto, V.; Oliveira, O. N., Jr. Immobilization of alcohol dehydrogenase in phospholipid Langmuir-Blodgett films to detect ethanol. Langmuir 2009, 25, 30573061.

(25) Giuffrida, M. C.; Dosio, F.; Castelli, F.; Sarpietro, M. G. Lipophilic prodrug of paclitaxel: Interaction with a dimyristoylphosphatidylcholine monolayer. Int. J. Pharm. 2014, 475, 624-631.

(26) Stillwell, W. Long-Range Membrane Properties. In $A n$ Introduction to Biological Membranes: Composition, Structure and Function; Elsevier Science: San Diego, 2016; pp 221-245.

(27) Krafft, M.-P. Controlling phospholipid self-assembly and film properties using highly fluorinated components - Fluorinated monolayers, vesicles, emulsions and microbubbles. Biochimie 2012, 94, 11-25.

(28) Corvis, Y.; Barzyk, W.; Brezesinski, G.; Mrabet, N.; Badis, M.; Hecht, S.; Rogalska, E. Interactions of a fungistatic antibiotic, griseofulvin, with phospholipid monolayers used as models of biological membranes. Langmuir 2006, 22, 7701-7711.

(29) Corvis, Y.; Brezesinski, G.; Rink, R.; Walcarius, A.; Van der Heyden, A.; Mutelet, F.; Rogalska, E. Analytical investigation of the interactions between SC3 hydrophobin and lipid layers: Elaborating of nanostructured matrixes for immobilizing redox systems. Anal. Chem. 2006, 78, 4850-4864.

(30) Gaines, G. L. Insoluble Monolayers at Liquid-Gas Interfaces; John Wiley and Sons: New York, 1966.

(31) Miller, C. A.; Neogi, P. Surfactants. In Interfacial Phenomena. Equilibrium and Dynamic Effects; Marcel Dekker Inc.: New York, 1985; pp 140-183.

(32) Bacon, K. J.; Barnes, G. T. Two-component monolayers. IV.The excess enthalpies and entropies of mixing in the octadecanoldocosyl sulfate system. J. Colloid Interface Sci. 1978, 67, 70-77.

(33) Korchowiec, B.; Paluch, M.; Corvis, Y.; Rogalska, E. A Langmuir film approach to elucidating interactions in lipid membranes: 1,2-dipalmitoyl-sn-glycero-3-phosphoethanolamine/cholesterol/metal cation systems. Chem. Phys. Lipids 2006, 144, 127-136. 